\title{
Exploring the Charm Sector with CLEO-c
}

\author{
D. Urner \\ Cornell University, Wilson Lab, Ithaca NY 14853, USA
}

\begin{abstract}
The CLEO collaboration proposes to explore the charm sector starting early 2003. It is foreseen to collect on the order of 6 million $D \bar{D}$ pairs, $300000 D_{s} \bar{D}_{s}$ pairs at threshold and one billion $J / \psi$ decays. High precision charm data will enable us to validate upcoming Lattice QCD calculations that are expected to produce 1-3\% errors for some non-perturbative QCD quantities. These can then be used to improve the accuracy of CKM elements. The radiative $J / \psi$ decays will be the first high statistics data set well suited for meson spectroscopy between 1600 and $3000 \mathrm{MeV}$.
\end{abstract}

\section{INTRODUCTION}

Let's start with a lofty goal: We strive for the mastery of a non-perturbative strongly coupled theory: QCD. Couplings in field theory do not typically have to be weak. Indeed, strong interactions are the expected phenomena if one reaches beyond the Standard Model. It will therefore be of great benefit if we can understand the effects of strong couplings in QCD. High precision predictions of QCD will also remove road blocks for many weak and flavor physics measurements.

Lattice QCD has matured over the last decade. We finally can expect the first nonperturbative QCD results with 1-3\% errors. CLEO-c will provide crucial data in a timely fashion to validate them and help guide the theory on its long way from easier predictions to a full understanding of non-perturbative QCD effects. This will result, for example, in improved measurements of $V_{c s}$ and $V_{c d}$ at the $1 \%$ level. CLEO-c data will also provide a large number of basic measurements needed in heavy flavor physics and future efforts in understanding physics beyond the standard model. A detailed description can be found in [1].

\section{Data Sets}

We plan to acquire the CLEO-c data in a 3 year program. The use of present and future CLEO data sets will be considered in the course of this paper. The expected size of the data sets are shown in Table 1.

CLEO-c intends to accumulate $30 \times 10^{6}$ events at $\psi^{\prime \prime}$ and about $1.5 \times 10^{6}$ events at $\psi(4140)$. The number of expected $D_{s} \bar{D}_{s}$ events is uncertain within a factor of two because of conflicting earlier measurements. This will be clarified with an early scan, which will determine the point of largest $D_{s} \bar{D}_{s}$ production. We expect to collect a total of about one billion $J / \psi$ events. Smaller data sets are considered at the $\tau \tau$ threshold (3557 $\mathrm{MeV})$ at the $\psi^{\prime}(3686)$ at $\Lambda_{c} \bar{\Lambda}_{c}$ threshold $(5200 \mathrm{MeV})$ and a scan over the full $3-7 \mathrm{GeV}$ 
TABLE 1. Size of datasets considered in the discussion of this paper. The data at the $\Upsilon(4 \mathrm{~S})$ represents the CLEOII and CLEOIII data sets. The data sets at the narrow $\Upsilon$ resonances are taken just prior to CLEO-c.

\begin{tabular}{lcc}
\hline Center of mass energy & Luminosity & Decays/Physics \\
\hline$\Upsilon(4 \mathrm{~S})(10580)$ & $24 \mathrm{fb}^{-1}$ & 2-photon physics \\
\hline$\Upsilon(3 \mathrm{~S})(10355)$ & $1 \mathrm{fb}^{-1}$ & $\eta_{b}$ \\
$\Upsilon(1 \mathrm{~S})(9460)$ & $1 \mathrm{fb}^{-1}$ & meson spectroscopy \\
$\Upsilon(2 \mathrm{~S})(10023)$ & $1 \mathrm{fb}^{-1}$ & \\
\hline$\psi^{\prime \prime}(3770)$ & $3 \mathrm{fb}^{-1}$ & $6 \times 10^{6} D \bar{D}$ \\
$\psi(4140)$ & $3 \mathrm{fb}^{-1}$ & $3.0 \times 10^{5} D_{s} \bar{D}_{s}$ \\
$J / \psi(3097)$ & $1 \mathrm{fb}^{-1}$ & $6.0 \times 10^{7}$ radiative $J / \psi$ decays \\
\hline
\end{tabular}

region.

In 2002, before lowering the energy to the charm sector, CLEO plans to collect data at the three narrow $1 S, 2 S$ and $3 S \Upsilon$ resonances with an integrated luminosity of about $1 \mathrm{fb}^{-1}$ each.

The CLEOII and CLEOIII data sets gathered at the $\Upsilon(4 S)$ contain a large number of 2-photon events.

\section{Accelerator: Modifications to CESR}

For the upgrade to CLEO III new superconducting quadrupoles for the final focusing system were built. They prove to be crucial, since they enable us to lower the beam energy and run in the region of the charm system. Accelerators find that the luminosity typically scales at best with $\mathrm{L} \sim \mathrm{E}_{b}^{4}$. This behavior can be changed if one introduces wigglers, which will cool the beam transversely to ideally a linear correlation of luminosity and beam energy. We plan to build 14 superconducting wiggler modules, each having $1.3 \mathrm{~m}$ of length, a peak field of $2 \mathrm{~T}$, and a $40 \mathrm{~cm}$ period. A 3-pole test module is shown in figure 1. The projected beam spread at the $\mathrm{J} / \psi$ will be about: $\Delta \mathrm{E}_{b} \sim 1.2 \mathrm{MeV}$. The expected machine performance is shown in Table 2.

TABLE 2. Expected machine performance for CLEO-c.

\begin{tabular}{lc}
\hline$\sqrt{s}$ & $\mathrm{~L}\left(10^{32} \mathrm{~cm}^{-2} \mathrm{~s}^{-1}\right)$ \\
\hline $4.1 \mathrm{GeV}$ & 3.6 \\
$3.77 \mathrm{GeV}$ & 3.0 \\
$3.1 \mathrm{GeV}$ & 2.0
\end{tabular}




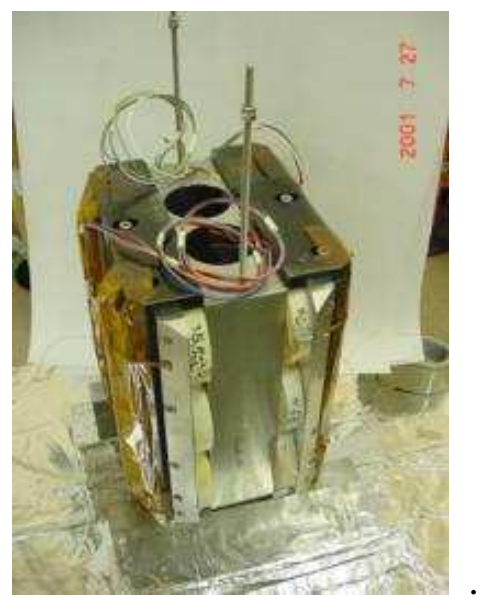

FIGURE 1. A 3 pole test module for the super conducting wigglers needed in the upgrade to CLEO-c

\section{Detector: CLEO III becomes CLEO-c}

The CLEO III detector is a wonderful detector to study the charm system. The tracking system and the calorimeter cover $93 \%$ of the solid angle, while the ring-imaging Cerenkov counter (RICH) covers $83 \%$ of the solid angle.

The tracking system consists of the main drift chamber [2] for which we find a hit resolution of $88 \mu \mathrm{m}$. The 4 layer silicon detector [3] has prematurely degraded, which is observed as a dramatic efficiency loss in the sensors signals. The origin of this problem is yet unknown. Cornell is building a 6-layer high angle stereo drift chamber to replace the silicon detector. For low momentum tracks, such as those typically generated when running at $\mathrm{J} / \psi$ energies, the performance of this drift chamber is comparable to a silicon vertex detector, because multiple scattering is the dominant contribution to the track resolution. We plan to run with a reduced $\mathrm{B}$ field of $1 \mathrm{~T}$ (1.5 Tesla for $\Upsilon$ running). That leads to a resolution of $0.35 \%$ at $1 \mathrm{GeV}$ for charged tracks.

The calorimeter [4] consists of 7800 Cesium Iodide crystals and measures the photons with a $\frac{\sigma_{E}}{E}=2 \%$ at $1 \mathrm{GeV}$ and $4 \%$ at $100 \mathrm{MeV}$.

Particle identification can be done with $\mathrm{dE} / \mathrm{dx}$ with a resolution of $5.7 \%$ for minimum ionizing pions. A ring imaging Čerenkov counter [5] has been installed with the CLEOIII upgrade. It has been shown to perform excellently. It covers $83 \%$ of the solid angle. For $0.9 \mathrm{GeV}$ particles the kaons are identified with $87 \%$ efficiency and a pion fake rate of $0.2 \%$.

Data from the drift chamber and the calorimeter are used in the trigger. It is pipelined with a latency of $2.5 \mu \mathrm{s}$. The trigger is fully programmable and can easily be adapted to the new event signatures.

The data acquisition system can accept hardware triggers up to $1 \mathrm{kHz}$ and is designed to write data to tape with a speed of about $300 \mathrm{~Hz}$. The event size is $25 \mathrm{kB}$ and the data throughput is $6 \mathrm{Mb}$. This means that the data acquisition infrastructure will be able to handle the large data rates at the $\mathrm{J} / \psi$ peak. 

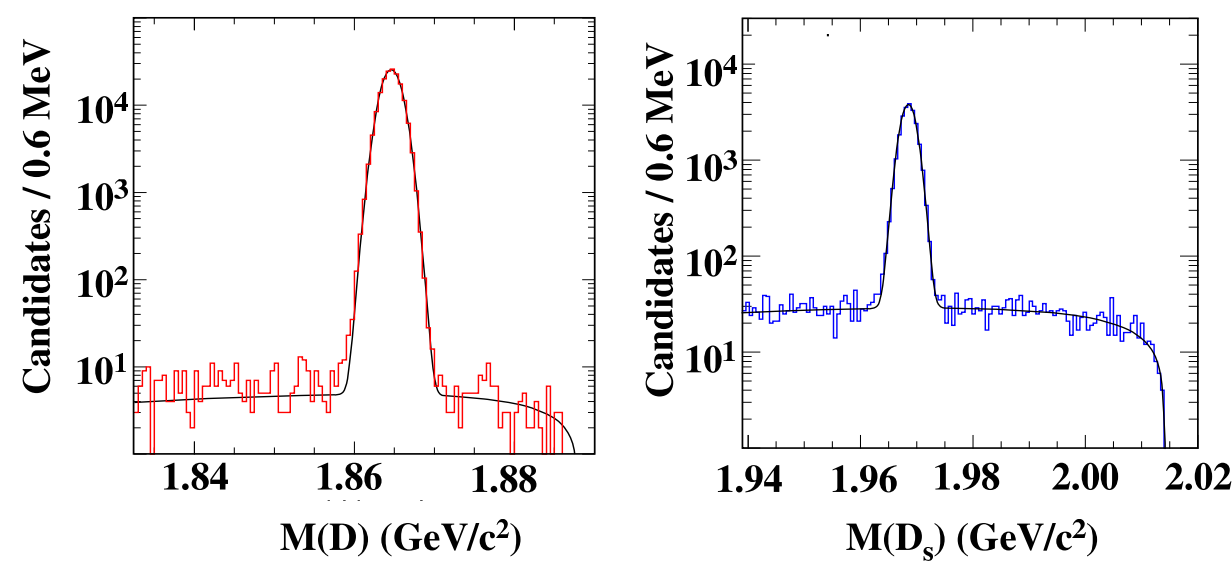

FIGURE 2. MC events equivalent to $1 \mathrm{fb}^{-1}$ of data. Left: $\mathrm{D} \rightarrow \mathrm{K} \pi$ tags. The width of the $\mathrm{D}$ peak is 1.3 $\mathrm{MeV} / \mathrm{c}^{2}$. Right: $\mathrm{D}_{s} \rightarrow \mathrm{KK} \pi$ tags. The width of the $\mathrm{D}_{s}$ peak is $1.4 \mathrm{MeV} / \mathrm{c}^{2}$.

\section{RUNNING AT THRESHOLD}

There are important advantages to running at the open flavor thresholds. Large cross sections mean the data can be acquired in one run period rather than over many years. The multiplicity of particles in the final state is smaller, which reduces backgrounds and increases efficiencies. We expect very clean data samples. Single tag events can be used to constrain $v$ reconstruction, double tags will be used for hadronic measurements. We anticipate 6 million $\mathrm{D}$ tags and $300,000 \mathrm{D}_{s}$ tags. The signal-to-background ratio for the $\mathrm{D} \rightarrow \mathrm{K} \pi$ tag is estimated to be $\mathrm{S} / \mathrm{B}: 5000$ and for $\mathrm{D}_{s} \rightarrow \mathrm{KK} \pi$ tag: $\mathrm{S} / \mathrm{B} \sim 100$, (see Figure 2). Many of the analyses can profit from using kinematic constraints at threshold. This can, for example, lead to a reduced systematic error if one can forego lepton identification. Further advantages are that the initial states are pure (no fragmentation) and that the D's are coherently produced.

\section{Absolute Hadronic Charmed Hadron Branching Fractions}

Absolute branching fractions are important since, for a lot of analyses at the highest energies as well as in the $B$-system, an inaccurate knowledge of $\mathrm{D}, \mathrm{D}_{s}, \ldots$ decays can result in large systematic errors. Using double-tagged events at threshold leaves only major systematic error contributions from efficiency uncertainties in the tracks and showers. An overview of the expected results is shown in Table 3.

In the case of $\mathrm{D}$ decays the statistics are high enough that we can concentrate on the most simple $\mathrm{D}$ decays. For one $\mathrm{fb}^{-1}$ we expect $1500 \mathrm{D}^{0} \rightarrow \mathrm{K}^{+} \pi^{-}$events with no background and $8446 \mathrm{D}^{+} \rightarrow \mathrm{K}^{-} \pi^{+} \pi^{+}$with 25 background events. For $\mathrm{D}_{s}$, the decays into $\mathrm{K}^{-} \mathrm{K}^{+} \pi^{-}, \mathrm{K}^{-} \mathrm{K}^{+} \pi^{-} \pi^{0}, \eta \pi^{-}, \eta \rho^{-}$, and $\eta^{\prime} \pi^{-}$were considered and combinations with less than $20 \%$ background were used to generate the numbers shown in Table 3.

The charmed baryon resonances measured with collisions at a large center of mass are typically presented as branching ratios with respect to the $\Lambda_{c}^{+} \rightarrow \mathrm{pK}^{-} \pi^{+}$decay. With 
TABLE 3. Total number of double tag events and precision on absolute charm branching fractions, assuming $3 \mathrm{fb}^{-1}$ data at $D \bar{D}, D_{s} \bar{D}_{s}$ thresholds and $1 \mathrm{fb}^{-1}$ at $\Lambda_{c} \bar{\Lambda}_{c}$ threshold

\begin{tabular}{lccccc}
\hline Particle & \# of double tags & $\begin{array}{c}\text { Statistical } \\
\text { Error }\end{array}$ & $\begin{array}{c}\text { Systematic } \\
\text { Error }\end{array}$ & $\begin{array}{c}\text { Background } \\
\text { Error }\end{array}$ & $\begin{array}{c}\text { Total } \\
\text { Error }\end{array}$ \\
\hline $\mathrm{D}^{0}$ & 53,000 & $0.4 \%$ & $0.4 \%$ & $0.06 \%$ & $0.6 \%$ \\
$\mathrm{D}^{+}$ & 60,000 & $0.4 \%$ & $0.6 \%$ & $0.10 \%$ & $0.7 \%$ \\
$\mathrm{D}_{s}^{+}$ & 6,000 & $1.3 \%$ & $1.1 \%$ & $0.90 \%$ & $1.9 \%$ \\
$\Lambda_{c}$ & 17,000 & $\sim 4 \%$ & small & small & $\sim 4 \%$
\end{tabular}

one $\mathrm{fb}^{-1}$ at the threshold $(4.6 \mathrm{GeV})$, one expects about $500 \Lambda_{c} \bar{\Lambda}_{c}$ double-tagged events, a rough estimate since neither the cross section nor the $\Lambda_{c}^{+} \rightarrow \mathrm{pK}^{-} \pi^{+}$branching fraction are well known.

\section{Meson Decay Constant}

The hadronic physics for leptonic decays of the $\mathrm{D}_{q}$ and $\mathrm{B}_{q}$ mesons is encapsulated in single non-perturbative QCD parameters $\mathrm{f}_{q}$. Given the knowledge of their values, one can extract $\left|\mathrm{V}_{c s}\right|,\left|\mathrm{V}_{c d}\right|,\left|\mathrm{V}_{t s}\right|$ and $\left|\mathrm{V}_{t d}\right|$. Our current knowledge of the uncertainty in $\mathrm{f}_{D_{s}}$ and $\mathrm{f}_{D}$ is $35 \%$ and $100 \%$, respectively, while $\mathrm{f}_{B}$ and $\mathrm{f}_{B_{S}}$ will not be measured in the foreseeable future. Lattice QCD should, however, be able to calculate the ratio of $\frac{f_{B}}{f_{D}}$ very accurately, so that the determination of $\mathrm{f}_{D}$ will indirectly help in the extraction of $\left|\mathrm{V}_{t s}\right|$ and $\left|\mathrm{V}_{t d}\right|$.

The leptonic $\mathrm{D}$ and $\mathrm{D}_{s}$ decay branching fractions are sizable and enable a direct determination of the charm meson decay constant from the measurement of $\mathrm{D}^{+} \rightarrow \mu^{+} \mathrm{v}$, $\mathrm{D}_{s}^{+} \rightarrow \mu^{+} \mathrm{v}$, and $\mathrm{D}_{s}^{+} \rightarrow \tau^{+} \mathrm{v}$. If one assumes unitarity of the CKM matrix to constrain the values of $\left|\mathrm{V}_{c d}\right|$ and $\left|\mathrm{V}_{c s}\right|$ one can measure $\frac{\delta f_{D_{S}}}{f_{D_{S}}}=2.1 \%$ and $\frac{\delta f_{D}}{f_{D}}=2.6 \%$.

\section{Semileptonic Form Factors and Determination of $\left|\mathbf{V}_{c s}\right|$ and $\left|\mathbf{V}_{c d}\right|$}

The semileptonic from factors $\left|f_{+}\left(q^{2}\right)\right|^{2}$ encapsulate the hadronic physics of semileptonic decays

$$
\frac{d \Gamma}{d q^{3}}=\frac{G_{F}^{2}}{24 \pi^{3}}\left|V_{c s}\right|^{2} p_{K}^{3}\left|f_{+}\left(q^{2}\right)\right|^{2}
$$

in the example of a $c \rightarrow s$ transition. If we again assume 3 generation unitarity one can extract the semileptonic form factors from processes like $\mathrm{D}^{0} \rightarrow \pi \mathrm{e} v$ or $\mathrm{D}^{+} \rightarrow \mathrm{K}^{* 0} \mathrm{e}^{+} \mathrm{v}$. The estimated precision for the parameters of the pseudoscalar to pseudoscalar and pseudoscalar to vector form factors are shown in Table 4. 
TABLE 4. This table contains uncertainties on the branching fractions for several $\mathrm{D}$ and $\mathrm{D}_{s}$ decay modes and precision of semileptonic form factor parameters.

\begin{tabular}{lcccccc}
$\begin{array}{l}\text { Decay } \\
\text { Mode }\end{array}$ & $\begin{array}{c}\text { PDG2000 } \\
(\delta \mathrm{B} / \mathrm{B} \%)\end{array}$ & $\begin{array}{c}\text { CLEO-c } \\
(\delta \mathrm{B} / \mathrm{B} \%)\end{array}$ & $\begin{array}{c}\text { Form Factor } \\
\text { Type }\end{array}$ & $\begin{array}{c}\text { Form Factor } \\
\text { Parameter }\end{array}$ & $\begin{array}{c}\text { expected } \\
\text { Precision }\end{array}$ & $\begin{array}{c}\text { CKM } \\
\text { Element }\end{array}$ \\
\hline $\mathrm{D}^{0} \rightarrow \mathrm{K}^{-} \mathrm{e}^{+} \mathrm{v}$ & 5 & 0.4 & & & $\left|V_{c s}\right|$ \\
$\mathrm{D}_{s}^{+} \rightarrow \phi^{-} \mathrm{e}^{+} v$ & 25 & 3.1 & & & $\left|V_{c s}\right|$ \\
$\mathrm{D}^{+} \rightarrow \pi^{0} \mathrm{e}^{+} \mathrm{v}$ & 48 & 2.0 & & & $\left|V_{c d}\right|$ \\
$\mathrm{D}^{0} \rightarrow \pi^{-} \mathrm{e}^{+} \mathrm{v}$ & 16 & 1.0 & $\mathrm{PS} \rightarrow \mathrm{PS}$ & $\delta \mathrm{f}_{+}(0) / \mathrm{f}_{+}(0)$ & $\sim 1 \%$ & $\left|V_{c d}\right|$ \\
& & & & slope & $\sim 4 \%$ & \\
$\mathrm{D}^{+} \rightarrow \bar{K}^{* 0} \mathrm{e}^{+} v$ & 9 & 0.6 & $\mathrm{PS} \rightarrow \mathrm{V}$ & $\delta \mathrm{A}_{1}(0) / \mathrm{A}_{1}(0)$ & $\sim 2 \%$ & $\left|V_{c s}\right|$ \\
& & & & $\delta \mathrm{A}_{2}(0) / \mathrm{A}_{2}(0)$ & $\sim 5 \%$ & \\
& & & & $\delta \mathrm{V}(0) / \mathrm{V}(0)$ & $\sim 5 \%$ &
\end{tabular}

$\left|\mathrm{V}_{c d}\right|$ is related to the decay rate $\Gamma$, which we can calculate from the absolute branching ratio $B\left(\mathrm{D}^{0} \rightarrow \mathrm{K}^{-} \mathrm{e}^{+} \mathrm{v}\right)$ and the mean lifetime $\tau_{D^{0}}$ as:

$$
\Gamma\left(D^{0} \rightarrow K^{-} e^{+} v\right)=\frac{B\left(D^{0} \rightarrow K^{-} e^{+} v\right)}{\tau_{D^{0}}}=T_{d}\left|V_{c s}\right|^{2}
$$

$\mathrm{T}_{d}$ is taken from theory and requires the knowledge of the PS to PS semileptonic form factor. We can expect lattice QCD calculations with an error of $\delta \mathrm{T}_{s} / \mathrm{T}_{s}=3 \%$ within a few years as discussed further below. This will result in a precision for $\left|\mathrm{V}_{c d}\right|$ of $1.7 \%$. In a similar way, $\left|\mathrm{V}_{c s}\right|$ can be determined with a precision of $1.6 \%$. Both CKM matrix elements can also be extracted from leptonic decays. Combining leptonic and semileptonic measurements we expect final precisions of $1.4 \%$ and $1.1 \%$ on $\left|\mathrm{V}_{c d}\right|$ and $\left|\mathrm{V}_{c s}\right|$, respectively.

\section{MAPPING OUT THE $\Upsilon$ AND $J / \psi$ SYSTEMS}

Starting late fall 2001 CLEO will collect about $1 \mathrm{fb}^{-1}$ on each of the $\Upsilon(1 \mathrm{~S})$ - $(3 \mathrm{~S})$ resonances. Most present theories [6] indicate that the ground state of the $\Upsilon$ system, the $\eta_{b}$, could be discovered via the hindered M1 transition from the $\Upsilon(3 S)$ state. One also would expect to see the decay $\Upsilon(3 \mathrm{~S}) \rightarrow \pi^{+} \pi^{-} \mathrm{h}_{b}$ [7]. If found, its large predicted decay branching fraction into $\eta_{b}$ of $50 \%$ opens a further avenue to observe the $\eta_{b}$.

CLEO also should be able to observe the $1^{3} \mathrm{D}_{J}$ states. The $b \bar{b}$ system is unique in that it has states with $\mathrm{L}=2$ that lie below the open-flavor threshold. We expect unquenched lattice calculations soon for the center of gravity of the triplets of both $\mathrm{D}$ states to about $\sim 5 \mathrm{MeV}$. Current theoretical calculations and our existing data suggest that we can expect 20 - 40 fully reconstructed events in the decay $\Upsilon(3 S) \rightarrow \gamma_{1} \chi_{b}^{\prime} \rightarrow \gamma_{1} \gamma_{2}\left({ }^{3} D_{J}\right)$ $\rightarrow \gamma_{1} \gamma_{2} \gamma_{3} \chi_{b} \rightarrow \gamma_{1} \gamma_{2} \gamma_{3} \gamma_{4} \Upsilon(1 S) \rightarrow \gamma_{1} \gamma_{2} \gamma_{3} \gamma_{4} l^{+} l^{-}$, which should enable the extraction of the center of gravity of the triplet of the $1^{3} \mathrm{D}_{J}$ state to about $3 \mathrm{MeV}$. From this, the mass of the lowest state can be predicted and a scan can directly establish its mass, gaining a measure of the S-D mixing in the $b \bar{b}$ system.

In the $\mathrm{J} / \psi$ system only very few states are measured with high precision. Although we know the ground state $\eta_{c}$, its width is measured very poorly. The $\eta_{c}^{\prime}$ and $h_{c}$ states need 
confirmation. The ${ }^{3,1} \mathrm{D}_{J}$ and $2^{3,1} \mathrm{P}_{J}$ states still have to be found. The region above the $D \bar{D}$ threshold is generally explored very little, despite the fact that a lot of interesting physics might be extracted if one could identify, for example, charmed hybrid states. CLEO-c will have to make a scan in order to find the energy with the largest $D_{s} \bar{D}_{s}$ decay rate. A more detailed scan between $3.6 \mathrm{GeV}$ and $5 \mathrm{GeV}$ is also considered.

\section{THE BRIGHT FUTURE OF LATTICE QCD}

Lattice QCD is a full implementation of QCD and can therefore in principle produce accurate results, also for the low energy phenomena that cannot be treated perturbatively. It has, however, failed to make good predictions with well understood errors because of technical difficulties [8]. In the last few years, some real breakthroughs have been achieved [9], so that we can expect some of the theoretically easier calculations to appear in the next few years with sound error estimates of $1-2 \%$.

In general, one was able to incorporate known features of QCD into the lattice calculations. Perturbation theory is used to describe short distance physics and connect the lattice to the continuum. Second order results lead to relative errors of $\mathrm{O}\left(\alpha_{s}^{3}\right)$. One would like to keep lattice spacing as large as possible in order to minimize computer time. Improved discretizations remove errors by adding correction terms. These and other improvements are required in order that one can finally get unquenched results, which include effects from $q \bar{q}$ loops. When this can be done with realistic d,u-quark masses, the errors on the results become quantifiable and errors of order $2 \%$ are achievable for many calculations. Examples are masses, decay constants, semileptonic form factors, and mixing amplitudes for $\mathrm{D}, \mathrm{D}_{s}, \mathrm{D}^{*}, \mathrm{D}_{s}^{*}, \mathrm{~B}, \mathrm{~B}_{s}, \mathrm{~B}^{*}, \mathrm{~B}_{s}^{*}$, and corresponding baryons; masses, leptonic widths, electromagnetic form factors, and mixing amplitudes for any meson in $\psi$ and $\Upsilon$ families below the open flavor threshold.

It should be stressed that lattice QCD can make all these predictions using only the quark masses and $\alpha_{s}$ as input parameters. Today there are not enough measurements in the one percent region for the quantities mentioned above. CLEO-c, however, will be able to provide most of them accurately enough, enabling us to validate the new lattice results and methods. Particularly the measurements in the $\psi$ and $\Upsilon$ region provide an excellent test ground. This validation is needed so that lattice QCD results can be trusted and used to increase the accuracy of theoretical predictions for many different aspects of physics.

\section{Impact of CLEO-c Results and Lattice QCD on Flavor Physics}

An immediate application of lattice QCD results by CLEO-c involves the extraction of $\left|\mathrm{V}_{c s}\right|$ and $\left|\mathrm{V}_{c d}\right|$ by the use of the meson decay form factor and the measurement of leptonic decay branching fractions of $\mathrm{D}$ and $\mathrm{D}_{s}$, as well as the semileptonic form factors and the measurement of the semileptonic decay branching fractions of $\mathrm{D}$ and $\mathrm{D}_{s}$, described above. 

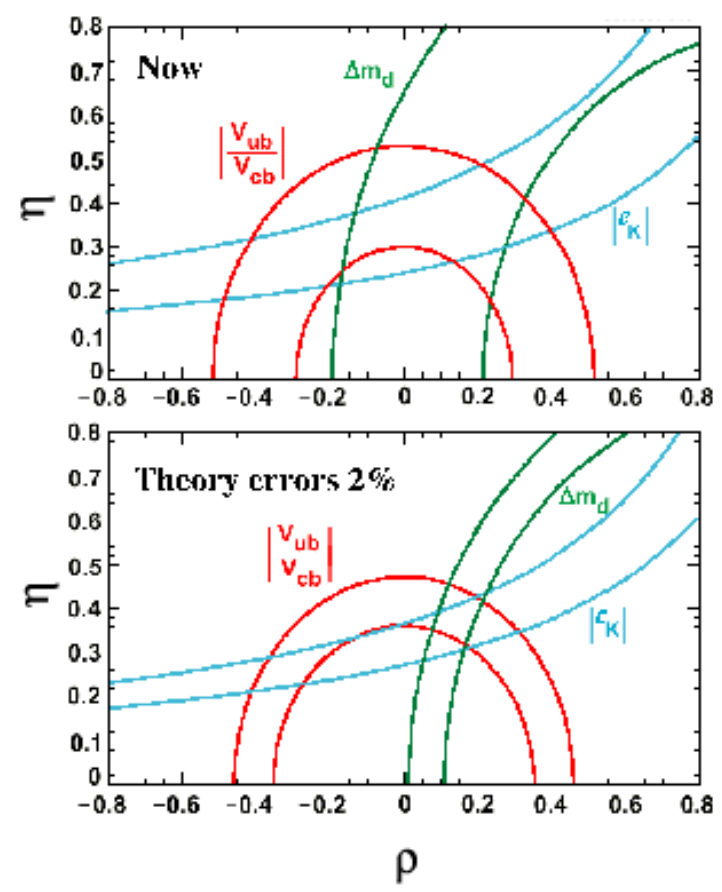

FIGURE 3. Above: Current situation taken from [10]. Below: Prediction on the limits using $2 \%$ errors on form factors and today's data.

Even before a full validation of lattice QCD, measurements of the D-meson form factor will result in better predictions of the B-meson form factor, which will tighten the constraints on the unitarity triangle from $B \bar{B}$ and $B_{s} \bar{B}_{s}$ mixing measurements. Using $\mathrm{SU}(3)$ and heavy quark symmetry the semileptonic form factor for the process $\mathrm{B} \rightarrow \pi \mathrm{e}^{+} \mathrm{v}$ can be predicted from the measurement of the semileptonic form factor extracted from the process $\mathrm{D}^{+} \rightarrow \mathrm{K}^{* 0} \mathrm{e}^{+} \mathrm{v}$, which will result in an improved $\left|\mathrm{V}_{u b}\right|$ determination.

Finally, the impact of $2 \%$ theoretical errors on form factors produced by a validated lattice QCD theory are shown in Figure 3 using today's measurements. It is obvious that only an increased bound from $\left|\mathrm{V}_{u b}\right|$ and $B \bar{B}$ mixing results together with the $\sin (2 \beta)$ measurements will be able to give a significant answer on the question if the unitarity triangle is indeed closed or if the effect of new physics is observed. The limits from the $\varepsilon_{K}$ measurement will also be improved considerably.

\section{MESON SPECTROSCOPY FROM $J / \psi$ DECAYS}

Lattice QCD will have answers for some questions in the near future, however there are many quantities that will remain difficult to calculate for some time. There are only some ideas on how to treat effects like mixing, or the inclusion of gluonic degrees of freedom. The important information that is needed to gain better understanding is the extraction of the relevant degrees of freedom of the strong interaction in the non-perturbative regime. 
A much better understanding of the light meson spectrum will be very helpful to gain this information and to guide the lattice.

An important advance will be if one could identify the $s \bar{s}$ states in the light meson sector. There are only a handful of $s \bar{s}$ states that are unambiguously known. However, they are needed in order to study complete nonets, and to discriminate $q \bar{q}$ against non$q \bar{q}$ resonances. CLEO-c will attempt to collect in the order of one billion $\mathrm{J} / \psi$ decays. This data set will contain some 60 million radiative $J / \psi$ decays. This will be the first data set with large statistics well suited to do meson spectroscopy in the region of 1.6 to $2.6 \mathrm{GeV}$. It should enable us to identify most of the $s \bar{s}$ states, since the initial state for radiative $\mathrm{J} / \psi$ decays is well defined and the CLEO detector will measure all final states simultaneously with $93 \%$ coverage in solid angle. Another way of identifying $s \bar{s}$ states is via $\psi$ and $\psi^{\prime} \rightarrow \mathrm{VF}$ flavor tagging. Lets say the vector state $\mathrm{V}$ might be reconstructed as $\omega, \phi$, or $\rho$ state. The particle $\mathrm{F}$ then will be dominantly a state of the same flavor as $\mathrm{V}$ due to the suppression of hair-pin diagrams.

It has been said many times that identifying the $q \bar{q}$ states is required first in order to find left over states. Further analysis is needed to determine if they are multiquark states, meson-antimeson molecules, hybrids (qqg) or glueballs. In practice however, these states can mix with the $q \bar{q}$ state. Current data seem to indicate that we have such a case in the scalar sector with the $\mathrm{f}_{0}(1400), \mathrm{f}_{0}(1500)$ and $\mathrm{f}_{0}(1710)$, which are thought to be the mixtures of two $q \bar{q}$ states and the glueball, although there are a fair number of other ideas on how to identify the scalars. Mixing to such a large degree does not have to be the typical case, and the idea of identifying the $q \bar{q}$ nonets should usually be possible.

The scalar sector requires special attention. The CLEO-c data set will be the only data set on the horizon that measures all three resonances simultaneously with high statistics and a well defined initial state. Furthermore, the scalars should be observed in $\Upsilon(1 S)$ decays, with lower statistics but well defined initial states. However the data set that will best reveal the nature of the scalar resonances is the 2-photon data collected in the $25 \mathrm{fb}^{-1}$ of CLEOII and CLEOIII data, since the coupling to glue is suppressed. Therefore the two photon partial width of the scalar states should give us a handle on their glue content.

One also expects hybrid states containing 2 quarks and a gluon as constituents. These are particularly interesting since there are hybrid states with quantum numbers not realized by $q \bar{q}$ states. The identification of a spectrum of hybrid states is very challenging, but has the reward to make unique measurements to test lattice QCD predictions. CLEO-c has limited access to hybrid production from $\chi_{c 1}$ decays, enabling us to measure $\mathrm{C}$-odd states as the $1^{-+}$exotic hybrid. Since the radiative decay fraction of the $\psi^{\prime} \rightarrow \chi_{c 1}$ is about $9 \%$, a sizeable number of $\chi_{c 1}$ decays should be recorded.

\section{A MULTITUDE OF OTHER EXCITING MEASUREMENTS}

There are a large number of other measurements possible, which are part of the very diverse CLEO-c program. There are just some highlights mentioned here. In $\tau$-physics we expect an improvement in the accuracy of our knowledge of the $\tau$-mass by a factor of three and of the Michel Parameter $\eta$ by a factor of four. We expect to detect many rare 
decays of the $\mathrm{D}^{0}$ and $\mathrm{D}^{+}$meson or set limits on the order of a few times $10^{-6}$. CLEO has the unique ability to perform an R-scan between 3-7 GeV, which would require about $150 \mathrm{pb}^{-1}$ of data and could be acquired within one week. This is of importance because it is expected that the $3-7 \mathrm{GeV}$ region will otherwise become the region contributing dominantly to uncertainties in the determination of $\alpha\left(\mathrm{M}_{Z}\right)$ and the hadronic contribution to $(\mathrm{g}-2)_{\mu}$.

\section{CONCLUSIONS}

The CLEO-c program has many exciting aspects. It is made unique, however, by the fact that it provides the data needed by an emerging lattice community to validate their new results to a level of a few percent. This combined effort should lead to a situation that many non-perturbative QCD calculations with well determined errors will be available in flavor physics and wherever they are needed to explore beyond the Standard Model.

\section{REFERENCES}

1. CLEO collaboration, CLNS 01/1742 at www.lns.cornell.edu/public/CLNS/2001/CLEO.html, CLEO-c and CESR-c: A New Frontier of Weak and Strong Interactions.

2. D. Peterson et al., Proceedings of th 8th Vienna Wire Chamber Conference, 19-23 February, Vienna, Austria, to be published in Nucl. Instrum. Methods A.

3. $\quad$ E. von Toerne et al., hep-ex/0103037, submitted to Nucl. Instrum. Methods A.

4. T. Hill, Nucl. Instrum. Methods A 418, 32 (1998).

5. M. Artuso et al. Nucl. Instrum. Meth. A 419, 577 (1998).

6. S. Godfrey and J. L. Rosner, EFI-01-10, April 2001, hep-ph/0104253, submitted to Physical Review D (Brief Reports).

7. Y.-P. Kuang and T.-M. Yan, Phys. Rev. D 24, 2874 (1981); M. B. Voloshin, Yad. Fiz. 43, 1571 (1986).

8. $\quad$ G. P. Lepage and P. B. Mackenzie, Phys. Rev. D 48, 2250 (1993).

9. $\quad$ See for example: M. Alford et al., Phys. Lett. B 361, 87 (1995); M. Luscher et al, Nucl. Phys. $B$ 478, 365 (1996).

10. A. Hocker et al., hep-ph/0104062. 